(C)2009 IEEE. Personal use of this material is permitted. However, permission to reprint/republish this material for advertising or promotional purposes or for creating new collective works for resale or redistribution to servers or lists, or to reuse any copyrighted component of this work in other works must be obtained from the IEEE. 


\section{Research Challenges in Applying Intelligent Wireless Sensors in the Oil, Gas and Resources Industries}

\author{
Alex Talevski \\ DEBII, Curtin University \\ Perth, Australia
}

\author{
Simon Carlsen \\ StatoilHydro ASA \\ Trondheim, Norway
}

\author{
Stig Petersen \\ SINTEF ICT \\ Trondheim, Norway
}

\begin{abstract}
The monitoring of oil, gas and resources plant performance and the operational environment through sensors allows for greater insight into potential safety problems and operational requirements. Such solutions promote a safe and healthy work environment for all stakeholders and optimized operations. Intelligent techniques and the monitoring of key historical operational properties can be used to realize certain characteristics and patterns in operation data. Such solutions may enhance operational visualization, foresight, forecasting and maintenance schedules for effective and efficient operation and maintenance. This optimizes plant safety, production, turnarounds, shutdowns and maintenance and improves error tolerance and recovery.
\end{abstract}

However, the development of robust devices that are able to perform in these remote and hostile requirements along with the intelligent solutions to structure, store, process and retrieve this information are difficult to realize. This paper investigates the use of wireless sensors and the related intelligent solutions in the oil, gas and resource industries.

\section{INTRODUCTION}

The monitoring of oil, gas and resources plant performance and the operational environment through sensors allows for greater insight into potential safety problems and operational requirements. Sensors may monitor pipeline pressure, flow, temperature, vibration, humidity, gas leaks, fire outbreaks, equipment condition and others. Sensor data is then used to make informed just in time decisions on platform operational conditions and performance. Through the use of intelligent techniques and the monitoring of key historical operational properties, sensor data may be used to realize certain characteristics and patterns in typical operations, promote a safe workplace and optimize production. Such solutions may enhance operational visualization, foresight, forecasting and maintenance schedules for effective and efficient operation and maintenance. Plant safety, production, turnarounds, shutdowns and maintenance, error tolerance and recovery are optimized.

Recent advances in wireless technology have enabled the development of wireless solutions capable of robust and reliable communication in harsh environments. International standards such as the IEEE $802.11 \mathrm{a} / \mathrm{b} / \mathrm{g} / \mathrm{n}$ [1][2][3][4] for wireless local area networks and the IEEE 802.15.4 [5] for low-rate wireless personal area networks, as well as numerous RFID (radio-frequency identification) specifications, have enabled applications such as wireless networking, sensing, monitoring, control, and asset tracking.
Wireless technology has the potential to be beneficial to the oil, gas and resources industries in a number of ways [6]. Eliminating the need for cables can contribute to;

- Reduced installation costs

- Reduced operating costs

- Reduced maintenance costs

- Speedy installation and removal in remote and hostile areas

- Mobile and temporary installations

The following six sections define the research challenges in applying intelligent wireless sensors, devices and networks in the oil, gas and resource industries;

- The use of robust wireless devices and networks in remote and hostile environments.

- The storage, structure and retrieval of the tsunami of wireless sensor data

- User interfaces for visualizing, configuring, monitoring and controlling wireless sensor networks and their outputs.

- Middleware to seamlessly integrate devices, with existing process control and monitoring systems and operating systemsand applications Convergence of technologies to deliver truly synchronized wireless voice, video and data.

- Wireless network and device security

\section{WireleSS DEVICES AND NETWORKS IN REMOTE AND HOSTILE ENVIRONMENTS}

Wireless devices are effectively self-contained. Totally wireless, battery operated devices, need to supply their own power either through a long life battery or through an alternative power generator such as solar energy. Hostile environments are not conducive to extended maintenance schedules. Therefore, such industries look for battery life greater than five years when such devices implement minute by minute updates.

Furthermore, wireless sensors in the oil and gas industry are typically deployed externally or on top of existing plant features. They need to be able to operate in highly hostile conditions which may include extreme temperature, wind, humidity, noise, dust, salt, rain etc. As wireless technologies are more susceptible to environmental changes than their wired counterparts, it is crucial to be able to quantify the 
expected and operational reliability and availability of the devices and their wireless link and/or network. Therefore, there are several challenges related to the introduction of such wireless devices in the oil, gas and resources industries. Such wireless devices must have the following properties;

- Restricted size, shape, construction and certification.

- Self contained. Ideally, when possible, devices should generate their own power or, contain battery packs with extended battery life of many years.

- Operate in a restrictive environment both in terms of radio noise and obstructions but also where certain restrictions on such radio devices are present (such as flammable areas).

- Operate in a hostile areas where environmental and platform conditions may be very harsh.

- Embedded platforms.

- Implement complex network algorithms with realtime requirements and adaptive routing protocols

- Contribute in a simplified ad-hoc and multi-hop network.

- Seamlessly integrate with existing IT solutions.

- Self re-configurable, dynamic and adaptive .

- Provide services within a dynamically changing system environment.

- Fault tolerance and recovery (self-healing, robust and reliable).

- Low maintenance.

As a result of very strict regulations on the installation of powered and networked sensors on oil, gas and resources platforms, the installations of additional sensors have, to date, been limited to what was installed when a plant was built. Using wireless solutions might be a simple and cost-efficient way of extending already existing wired plant networks. However, studies performed in [7] show that the required changes in factory and plant work processes might be the largest hindrance for the introduction of wireless technology in the oil, gas and resources industries. Furthermore, it has been noted that problems are typically experienced when the human factors are ignored in the adoption of new technology.

Wireless technology has the potential to be beneficial in many regards [6]. Eliminating the need for cables can contribute to reduced installation and operating costs, it enables installations in remote areas, and it allows for costefficient, temporary and mobile systems. The technical requirements for the deployment of wireless technology in the oil, gas and resources industries have been identified in [7]. As wireless technologies have developed, we are currently seeing the use of wireless sensors being installed on older end-of-lifecycle platforms in order to gain new insights and optimize production. This has obviously created great opportunities to improve operations but has also introduced numerous research and development challenges that are further discussed below.

\section{Storage And Retrieval of the Tsunami of Sensor} DATA

IP traffic will nearly double every two years through to 2012 [8]. Therefore, total IP traffic will increase by a factor of six from 2007 to 2012. High-definition video, audio and other high-resolution, high update streaming data such as that produced by cctv cameras and sensors over high-speed connections will be the main driver behind the $46 \%$ pa IP growth in the coming years. Such streaming data may now accounts for approximately one-quarter of all Intranet traffic. Streaming data grew from 12 percent in 2006 to 32 percent in 2008 [8]. The rapid growth of the use of high resolution devices on our networks has produced a significant challenge in dealing with such a tsunami of data.

Oil, gas and resources production plants may utilize 1000s of streaming sensors and cameras on their processing platforms in order to monitor operational conditions. These sensors are an integral part of the safe and optimal operation of such plants. These sensor produce significant amounts of unstructured and unclassified data that is simply used to momentary visualize plant conditions.

The oil and gas Industry has introduced requirements for the storage of historical process data. It is essential that sensor systems have sufficient network bandwidth, processing power and storage capacity to cater for the tsunami of data that is experienced. This information may be valuable for future reference and it may be possible to intelligently process this historical data in order to gain insight on typical plant operation characteristics and how they can be optimized. Therefore, it is essential that such data is appropriately captured, structured and stored.

Semantics is the study of the meaning in communication. It represents the definitions of the meaning of data elements, as opposed to the rules for encoding or representation. Semantics describe the relationship between the syntactical elements and the model of computation. It is used to define what entities mean with respect to their roles in a system. This includes capabilities, or features that are available within a system.

Ontologies are formal, explicit specifications of a shared semantic conceptualization that are machine-understandable and abstract models of consensual knowledge. They are concerned with all processes of component operation. They define concepts, abstractions, relationships and interactions as domain concepts and instantiations for manual or automated reasoning. These ontologies signify information which evolves to reflect the operational environment. Reaching such a formal consensus of understanding is of benefit in an environment that deals with self-contained, distributed and heterogeneous wireless sensors. In general the following system properties can be defined:

- Information and Communication - refers to the basic ability to gather and exchange information between the parties involved.

- Integrability - relates to the ability of sensors from 
different sources to mesh relatively seamlessly and the ability to integrate these sensors to build the final solution in a straightforward way.

- Coordination - focuses on scheduling and ordering tasks performed by these parties.

- Awareness and Cooperation - refer to the implicit knowledge of the operation process that is being performed and the state of the system.

An ontology framework enforces an agreement on how information should be organized, without losing any of the flexibility of allowing expansion through the following:

- Normative model - creates a extendible semantic model that can later be refined, and which allows semantic transformations between different contexts.

- $\quad$ Relationships - keep track of progress and monitor quality and productivity from different components.

- Consistency - provides unambiguous definitions for terms in the production system.

- Integration - by providing a normative model, integration of distributed and heterogeneous components can be accessed.

The integration of a number of (existing) ontologies for other domains can be used to augment the functionality of any framework. These ontologies provide a distributed workflow technique across multiple sites through;

- Process Ontology - develops a representation of the different types of processes involved in plant operation. Previously taxonomies for plant operation have been developed by Holland [9] and refined by Lastra [10]. These ideas were extended to an Ontology by Delamar [11].

- Component Ontology - provides a representation of all the components involved in the production operation activity at the different levels of granularity. As each large granularity component is likely to consist of subcomponents itself, it is important that these are all represented within the ontology.

- $\quad$ Product Ontology - represents the different types of products or units that will result from the operation activity. They involve classification of the products and its features. Here the relationships involved and the layout configurations are captured.

Each defined ontology conceptually represents the perceived domain knowledge through its concepts, attributes, taxonomies, relationships, and instances for plant operations.

Ontology systems may be built on top of Jena [10]. Developed by Hewlett-Packard, Jena 2.1 is a framework with the capacity of manipulating ontologies [11]. The ontology system provides navigating, querying, and manipulating functions. The design philosophy of the ontology system is to use the in-memory storage model and serialize it into a physical document stored in the ontology repository. The ontology system provides three services: navigating, querying and manipulating services. Ontology can be navigated for clarification or classification of certain concepts. Software agents can then automatically reason about the data stored in these repositories to optimise user interaction and/or system operation.

\section{Sensor Output Visualization, Control System USER INTERFACES AND REMOTE OPERATIONS}

Wireless systems need to be configured, monitored and controlled throughout their lifetime. In an oil, gas and resources facilities, it is desirable that these tasks can be performed by personnel who posses little understanding and knowledge of the underlying technology. Introducing wireless sensors in a facility should be transparent and straightforward process. If this is not the case, one would have to rely on technical experts to be brought on-site whenever parameters of the wireless systems have to be altered, or technical problems arise. This is particularly expensive and time consuming when plants are located in remote, offshore and/or hostile areas. The extended delay in having to transport a technical expert onsite may lead to a production stop or a facility shut down. This is simply not an option.

As a consequence, the user interfaces for wireless systems must be able to provide a simple and intuitive but powerful interface for advanced remote configuration, control and management. Creating such applications is a challenge, and it requires the software developers not only to have extensive expertise of the systems, but also to understand the psychological phenomena behind the art of creating good user interfaces.

Existing sensor output handling has outlined many deficiencies in information processing and presentation. System operators are often presented with fixed schematics of large amounts of data that must be processed quickly and decisively. However, the amount of information required to be digested is quite large and crucial decisions need to be made within a constrained timeframe. This can leave the system operator suffering from information overload when decisions have the greatest impact. This problem requires the following areas to be investigated in detail;

- Interpreting sensor messages

- Classification of output messages through the relationship between sensor outputs

- Separation of output categories

- Suppression of situation irrelevant output messages

- Combination of common messages

- Production of a timely, informed, detailed and powerful output message Define system impact following sensor output 


\section{DEVICE MiddleWARE}

Middleware is traditionally used as a bridge between operating systems (and similar low-level constructs) and components [12]. However, common middleware systems for traditional distributed systems, such as CORBA [13], and the IndustrialIT [14] framework developed by $\mathrm{ABB}$ for distributed industrial systems, are not capable of supporting the dynamic and adaptive environments found in the oil, gas and resources industries. New WSN systems are required to seamlessly integrate into the existing systems or be used to create completely new ones while considering the strict requirements of this environment. It is essential that both these processes are fast and easy to perform as a response to production requirements. This has lead to the research and development of middleware specifically designed to meet the challenges of dynamic and resource-constrained WSNs. A survey performed in [15], identifies the following challenging areas for WSN middleware:

- Managing limited power and resources

- Scalability, mobility and dynamic network topology

- Heterogeneity

- Dynamic network organization

- Real-world integration

- Data aggregation

- Quality of Service

- Security

Early WSN specific middleware, such as Autosec [16] and Impala [17], were designed for efficient use of the wireless sensor network. Recent research, such as the development of the MiLAN middleware for WSNs [18], concludes that for WSN applications it is not a viable approach to handle the network management independent of the needs of the application. A WSN middleware should be able to trade application performance for network cost, while still maintaining the separation between the policy specifying the reaction to a dynamic environment and the mechanisms to implement such a policy.

In order to solve the sensor integration problems, the supporting IT systems need to be simple and malleable. Research [19] has outlined the requirement for a servicebased approach. Service-based approaches achieve loose coupling among interacting systems where disparate components may interact using a common interaction protocol and certain architectural and protocol constraints. By abstracting a component's internals through an interface, components become well isolated and standardized. Such architectures define component location, integration, management, monitoring and security in a straightforward way. Tailorable systems may then be rapidly adapted in a straightforward way to satisfy the specialized and evolving requirements. Tailorable system architectures require specific tools and significant technological support. We have identified the following categories and features that must be investigated when developing tailorable WSN architectures;

\section{Composition}

- Context independence - Identifying the clear decompositions in such a system is a key to developing a hierarchical system architecture where applications are built from building blocks.

- Service Model - A service model ensures that disparate components which reside on different platforms, can interoperate.

\section{Interaction}

- Accessibility - Services must be accessible by clients that are implemented using different technologies, and are distributed over a network.

- Data Exchange - A data exchange model is a communication protocol that enables components to interact and transfer data in a standardized way.

- Location Transparency - To achieve location transparency and seamless interoperability, a piece of wrapping middleware code lies between components that make interactions transparent.

- Contracts - An interaction contract guarantees that an interface exists, and will provide its advertised services.

\section{Collaboration}

- Plug and Play - The use of stable, published interfaces enables assembly or integration of applications from disparate sources. In this way, components can be reconfigured, added, removed, or replaced after system deployment.

- Automation - The use of macros and scripts that are implemented to facilitate dynamic discovery, interaction and integration of reusable components at runtime [20][21].

\section{CONVERGENCE OF TECHNOLOGIES FOR SYNCHRONIZED VOICE, VIDEO AND DATA}

Oil, gas and resource production plants use a variety of sensors that produce a visual representation of the just-in-time plant operational state. Such sensors are particularly important when the platform functions are operated remotely. The use of sensor data supports the move from "Decision Support" operations to true "Remote Operations". Collaboration across disciplines, companies, organizational and geographical boundaries, can only be made possible by wireless real-time data and the related new work processes. Remote operations offer significant operational cost savings 
and efficient use of resources across multiple sites. Remote operations have become more and more popular in these industries. These processes take as many operational people as possible away from hazardous production platforms. This has significant benefits for employee health and safety, improves their livelihood and reduces time that they spend away from home. In-turn, operating costs are significantly reduced. There are even ongoing research projects in the oil and gas industry looking into the development of completely unmanned production platforms. For such a scheme to be successful, it is essential that cctv cameras, microphones, communications, control systems and other data that is transmitted is synchronized and converged.

In the IT\&T world, convergence refers to the move towards the use of a single united interaction medium and media as opposed to the many that we use today [22]. Such a solution enables communications services that are concurrently coupled with enterprise and internet data. The ability to visualize a concept via images, graphs, tables and procedures while communicating over the telephone greatly enhances such interactions. Interaction is more pleasing, meaningful, effective and efficient. Actions can be taken with greater precision and faster response to market drivers. Therefore, data and telecommunications convergence promises a wide range of possible solutions that will increase productivity and flexibility, reduce costs, and provide new opportunities and revenues for enterprises.

From an enterprise perspective, converged voice and data services and interaction mediums to its employees, partners and end customers is essential. The following features are required;

- Multimedia - Converged voice, video and data services and interaction mediums are required as a consequence of the increased flexibility that businesses demand [23]. Such a collaboration environment is more pleasing, meaningful, effective and efficient.

- Accessibility - The availability of converged communications and, enterprise and internet services via a variety of mobile devices is essential. Such devices (PC, PDA, Mobile Phone and others), voice/data transportation mediums and a variety of protocols and encodings must be considered.

- Feature Rich Services - The combination of major communication functions with services that access enterprise and internet data such as databases, applications and web services via multiple interfaces essential.

- Multi-Interface - Being able to visualize the context of a conversation greatly enhances interaction. Voice, video and data interfaces project service data and system/user interaction. System interaction should be interchangeably possible through the device interface (DTMF, keyboard, mouse, joystick etc) and/or through voice driven commands.
- Flexible and Adaptive Environment - A problem faced in developing a feature-rich, flexible and widely accessible solution is the service integration and reconfiguration issues occurring on the disparate layers of communications and computer services. Re-configurable software architectures promote simplified software evolution in complex environments.

\section{Wireless NeTWORK AND DEVICE SECURITY}

With the use of wireless networks and devices, new privacy and security threats are more prevalent. Such systems must implement strict encryption, transmitter authentication and data consistency validation. Wireless networks are susceptible to various security issues. In such sensitive commercial environments it is essential that security is assured from attacks such as;

- Accidental Association - refers to unintentional access to wireless networks where foreign computers or devices may inadvertently connect to an overlapping neighboring wireless networkwithout being aware that this is even happening $\mathrm{y}$. This still represents a significant security breach in proprietary network and may expose sensitive company systems and data.

- Malicious Association - is created when access to a network is obtained by hackers. This is typically performed through weak security measures and protocol loopholes. It may also be possible to lure computers to login to networks that impersonate the real thing by exploiting faults in the wireless protocol. By temporarily disrupting the response of a real network and simultaneously granting access to an impostor equivalent it is possible to involuntarily capture a user or group of users and transparently route communications through a central hacker point. This makes it possible to capture valid users, steal passwords and data, launch other attacks and install trojans.

- Identity Theft - Identity theft occurs when a hacker is able to listen-in to key user credential traffic and is able to use this information to impersonate an authorized computer and/or user.

- Man-in-the-Middle Attacks - Man-in-the-Middle Attacks use the Malicious Association techniques to gain access to a network and its users and transparently monitor passing traffic. If data is unencrypted or is easy to decipher then a hacker is given access to sensitive company information. A hacker may transparently listen to, remove and/or replace key network packets with others in order to provide misleading information.

- Denial of Service - A Denial-of-Service attack (DoS) attack occurs when a targeted access point or 
device is flooded with bogus protocol messages and data in an attempt to reduce or even suspend its responsiveness and ability to perform its regular functions. This is a very serious problem when wireless devices may be required to deliver time critical data. Jamming the wireless communication link utilizing dedicated jamming devices also falls into the Denial-of-Service category.

- Network Injection - A network injection attack makes use of access points that are exposed to nonfiltered or broadcast network traffic, by introducing bogus network configuration commands that may affect routers, switches, and intelligent hubs. The network devices may crash, shutdown, restart or even require reprogramming.

- Radio Interference - As more and more wireless communication devices utilize the license free portions of the frequency spectrum, in particular the ISM bands, friendly coexistence between the different systems and technologies is of greatest importance.

\section{CONCLUSION}

Sensors are able to monitor plant performance and the operational environment of oil, gas and resource production plants. When effectively deployed, this arrangement allows great insight into plant safety and performance. Well developed sensor solutions promote a safe and healthy workplace and simultaneously give the ability to optimize production.

Through the use of well engineered sensor networks that are suited to oil, gas and resource operations and intelligent processing techniques, sensor data can be used to realize certain characteristics and patterns in operation data. Such solutions may enhance operational visualization, foresight, forecasting and maintenance schedules for effective and efficient operation and maintenance. This optimizes plant safety, production, turnarounds, shutdowns and maintenance and improves error tolerance and recovery.

\section{REFERENCES}

[1] IEEE Computer Society, "IEEE 802.11-1999: Wireless LAN Medium Access Control (MAC) and Physical Layer (PHY) specifications", Standard, July 1999.

[2] IEEE Computer Society, "IEEE 802.11a-1999: Highspeed Physical Layer in the $5 \mathrm{GHz}$ Band", Standard, February 1999.

[3] IEEE Computer Society, "IEEE 802.11b-1999: Wireless LAN Medium Access Control (MAC) and Physical Layer (PHY) specifications: HigherSpeed Physical Layer Extension in the 2.4 GHz Band", Standard, October 1999.

[4] IEEE Computer Society, "IEEE 802.11g-2003: Wireless LAN Medium Access Control (MAC) and Physical Layer (PHY) specifications: Further Higher Data Rate Extension in the 2.4 GHz Band", Standard, June 2003.

[5] IEEE Computer Society, "IEEE 802.15.4-2006: Wireless Medium Access Control (MAC) and Physical Layer (PHY) Specifications for Low-Rate
Wireless Personal Area Networks (LR_WPANs)", Standard, December 2006.

[6] Petersen, S, et al, "Requirements, Drivers and Analysis of Wireless Sensor Networks for the Oil \& Gas Industry", In Proceedings of the 12th IEEE International Conference on Emerging Technologies and Factory Automation, Patras, Greece. September 2007.

[7] Petersen, S, et al, "A Survey of Wireless Technology for the Oil \& Gas Industry", In Proceedings of the SPE Intelligent Energy Conference, Amsterdam, Netherlands, February 2008.

[8] Cisco, "Visual Networking Index - Forecast and Methodology 20072012", On-line at: http://www.cisco.com/ (2009)

[9] Holland, N.J, Feminist Interpretations of Jacques Derrida Published by Penn State Press, 1997 ISBN 0271016353, 9780271016351

[10] Martinez Lastra, J.L, Colombo, A.W, "Domain Ontologies for Reasoning Machines in Factory Automation: Future Trends", On-line at: $\mathrm{ftp}: / / \mathrm{ftp}$.cordis.europa.eu/pub/ims/docs/3-7-colombo-lastra.pdf (2009)

[11] Delamare, le Deist, F., Winterton, J, What is Competence?, Human Resource Development International, Volume 8, Number 1, March 2005.

[12] Heinzelman, W.B., Murphy, A.L., Carvalho, H.S., and Perillo, M.A., "Middleware to Support Sensor Network Applications", IEEE Network, January 2004, pp 6-14.

[13] OMG, "Common Object Request Broker Architecture: Core Specification", Version 3.0.3, March 2004.

[14] Bratthall, L.G, et al, "Integrating Hundred's of Products through One Architecture - The Industrial IT Architecture", In Proceedings of the 24th International Conferenceon Software Engineering, Orlando, Florida, May 2002.

[15] Petersen, S, Carlsen, S, Talevski, A, "Industrial IT Revolution through Wireless Sensor Network Technologies", In Proceedigns of the ICST I.T. Revolutions Conference, Venice, Italy, December 2008

[16] Han, Q, Venkatasubramanian, N, "Autosec: An Integrated Middleware Framework for Dynamic Service Brokering", IEEE Distributed Systems Online, Volune 2, Number 7, 2001.

[17] Liu, T., and Martonosi, M., "Impala: A Middleware System for Managing Autonomic, Parallel Sensor Systems", In Proceedings of the ACM SIGPLAN Symposium on Principles and Practice of Parallel Programming, San Diego, California, June 2003.

[18] Murphy, A, Heinzelman, W, "MiLan: Middleware Linking Applications and Networks," Univ. of Rochester, tech. rep. TR-795, 2002.

[19] Schutte, R, "SOA is changing software", On-line at: http://www.gartner.com/resources/111900/111987/111987.pdf (2009).

[20] Chang, E, Annal, D, Grunta, F, "A Large Scale Distributed Object Architecture - CORBA \& COM for Real Time Systems", In Proceedings of the Third IEEE Conference on Object Oriented Real Time Distributed Systems, Newport Beach, California, March 2000.

[21] Annal, D, "NetCaptain Client Architecture", System Development Document, Philips Public Communications Pty. Ltd, Australia, August 1999.

[22] Talevski, A, Chang, E, "Reconfigurable Software Architecture for Voice Access to Data Services", In Proceedings of the Inaugural IEEE-IES Digital EcoSystems and Technologies Conference DEST '07, February 2007.

[23] Hui, M.C, Matthews, H.S, "Comparative analysis of traditional telephone and Voice-over-Internet Protocol (VoIP) systems", In Proceedings of the IEEE International Symposium on Electronics and the Environment, Scottsdale, USA, 2004

[24] Dillon, T.S, Niebur, D, Neural Networks Applications in Power Systems. London: CRL Publishing Ltd, 1996.

[25] Dillon, T S, Morsztyn, K and Phua, K, "Short-Term Load Foercasting Using Adaptive Pattern Recognition and Self-Organising Techniques", In Proceedings of the Power System Conputation Conference, Cambridge, UK, March 1975

[26] Ruiz, P.A, Gross, G, "Short-Term Resource Adequacy in Electricity Market Design", IEEE Transactions on Power Systems, Volume 23, Issue 3, August 2008, pp: 916-926

[27] Moghram, I, Rahman, S, "Analysis and Evaluation of Five Short-Term Load Forecasting Techniques", IEEE Transactions on PWRS, Volume 4, Issue 4, November 1989, pp: 1484-1491

[28] Ferreira, V.H, Alves da Silva, A.P, "Toward Estimating Autonomous Neural Network-Based Electric Load Forecasters", IEEE Transactions on Power Systems, Volume 22, Issue 4, November 2007, pp: 1554-1562 [29] Prince, W.R, "A Survey of Current Operational Problems", IEEE Power Engineering Review, Volume 9, Issue 11, November 1989, pp: 43-43

[30] Khosla, R, Dillon, T.S “A Neuro-Ezpert Systems for Power System Problems”, International Journal of Engineering Intelligent Systems, Vol 2 (1994) 